\title{
Renal outcomes of laparoscopic versus open surgery in patients with rectal cancer: a propensity score analysis
}

\author{
Jin Hyuk Paek ${ }^{1}$, Sung II Kang ${ }^{2}$, Jiwon Ryu ${ }^{3}$, Sung Yoon Lim ${ }^{3}$, Ji Young Ryu ${ }^{3}$, Hyung Eun Son ${ }^{3}$, Jong Cheol Jeong ${ }^{3}$, \\ Ho Jun $\mathrm{Chin}^{3}$, Ki Young $\mathrm{Na}^{3}$, Dong-Wan Chae ${ }^{3}$, Sung-Bum Kang ${ }^{4}$, Sejoong $\mathrm{Kim}^{3}$ \\ ${ }^{1}$ Department of Internal Medicine, Keimyung University School of Medicine, Daegu, Republic of Korea \\ ${ }^{2}$ Department of Surgery, Yeungnam University College of Medicine, Daegu, Republic of Korea \\ ${ }^{3}$ Department of Internal Medicine, Seoul National University Bundang Hospital, Seongnam, Republic of Korea \\ ${ }^{4}$ Department of Surgery, Seoul National University Bundang Hospital, Seongnam, Republic of Korea
}

Background: A laparoscopic approach is widely used in abdominal surgery. Although several studies have compared surgical and oncological outcomes between laparoscopic surgery (LS) and open surgery (OS) in rectal cancer patients, there have been few studies on postoperative renal outcomes.

Methods: We conducted a retrospective cohort study involving 1,633 patients who underwent rectal cancer surgery between 2003 and 2017. Postoperative acute kidney injury (AKI) was diagnosed according to the serum creatinine criteria of the Kidney Disease: Improving Global Outcomes classification.

Results: Among the 1,633 patients, 1,072 (65.6\%) underwent LS. After matching propensity scores, 395 patients were included in each group. The incidence of postoperative AKI in the LS group was significantly lower than in the OS group (9.9\% vs. 15.9\%; $p=$ 0.01). Operation time, estimated blood loss, and incidence of transfusion in the LS group were significantly lower than those in the OS group. Cox proportional hazard models revealed that LS was associated with decreased risk of postoperative AKI (hazard ratio [HR], 0.599; 95\% confidence interval [Cl], 0.402-0.893; $p=0.01$ ) and postoperative transfusion was associated with increased risk of AKI (HR, 2.495; 95\% Cl, 1.529-4.072; $p$ 0.001). In the subgroup analysis, the incidence of postoperative AKI in patients with middle or high rectal cancer who underwent LS was much lower than in those who underwent OS (HR, 0.373; 95\% Cl, 0.197-0.705; $p=0.002$ ).

Conclusion: This study showed that LS may have a favorable effect on the development of postoperative AKI in patients with rectal cancer.

Keywords: Acute kidney injury, Colorectal surgery, Laparoscopy, Rectal neoplasms

Received: January 1, 2021; Revised: May 31, 2021; Accepted: June 27, 2021

Editor: Jong Hyun Jhee, Yonsei University, Seoul, Republic of Korea

Correspondence: Sejoong Kim

Department of Internal Medicine, Seoul National University Bundang Hospital, 82 Gumi-ro 173beon-gil, Bundang-gu, Seongnam 13620, Republic of Korea. E-mail: sejoong2@snu.ac.krt

ORCID: https://orcid.org/0000-0002-7238-9962

Jin Hyuk Paek, Sung Il Kang, and Jiwon Ryu contributed equally to this study as the co-first authors.

Copyright (C) 2021 by The Korean Society of Nephrology

(a) This is an Open Access article distributed under the terms of the Creative Commons Attribution Non-Commercial and No Derivatives License (http:// creativecommons.org/licenses/by-nc-nd/4.0/) which permits unrestricted non-commercial use, distribution of the material without any modifications, and reproduction in any medium, provided the original works properly cited. 


\section{Introduction}

Acute kidney injury (AKI) is a clinical syndrome that affects kidney structure and function. It is characterized by abrupt loss of kidney function [1]. AKI is a serious complication that increases economic costs as well as the risk of mortality and morbidity [2,3]. The incidence of AKI among hospitalized patients and intensive care unit patients has been reported to be $3 \%-7 \%$ and $22 \%$, respectively [3-5]. AKI is a common postoperative complication and postoperative AKI accounts for approximately $30 \%$ to $40 \%$ of in-hospital AKI [6]. Postoperative AKI is associated with prolonged hospital stay, increased risk of mortality, and progression to chronic kidney disease $[7,8]$.

Laparoscopic surgery (LS) is increasingly being performed for the treatment of colorectal cancer in several centers. Its advantages include less pain, reduced intraoperative blood loss, and shorter recovery time [9]. However, there are concerns about increased intraabdominal pressure resulting from pneumoperitoneum. In patients with liver resection surgery, postoperative AKI was more common in the open surgery (OS) group than in the LS group [10]. Another study involving patients who underwent pylorus-preserving pancreaticoduodenectomy revealed that there were no significant differences in the incidence of AKI between LS and OS groups [11]. Although several studies have compared surgical and oncological outcomes between LS and OS in rectal cancer patients, there are few studies focusing on postoperative renal outcomes [12,13].

Therefore, we conducted a large retrospective cohort study to compare renal outcomes following LS and OS in patients with rectal cancer. We hypothesized that LS might have a positive effect on postoperative renal outcomes in rectal cancer patients. We performed propensity score matching analysis to minimize confounding biases.

\section{Methods}

\section{Study design and population}

This retrospective study included all patients who underwent rectal cancer surgery at the Seoul National University Bundang Hospital (Seongnam, Korea) from May 2003 to May 2017. Among the 1,678 patients identified, 45 were excluded for the following reasons: underwent local excision
( $n=31)$ or emergency surgery $(n=4)$; had end-stage renal disease ( $(n=4)$; and had insufficient data $(n=6)$. Finally, 1,633 patients were enrolled in this study (Fig. 1).

The Institutional Review Board (IRB) of Seoul National University Bundang Hospital approved this study (No. B-1707/411-105). The requirement of written informed consent was waived by the IRB because of the retrospective nature of this study.

\section{Data collection and definitions}

Electronic medical records of the study population were reviewed to retrieve patients' baseline characteristics, laboratory findings, and intraoperative data. Age, sex, body mass index [14], comorbidities (hypertension and diabetes mellitus), stage of malignancy, neoadjuvant chemotherapy, previous operation history, American Society of Anesthesiologists (ASA) physical status (PS) classification [15], and years of surgery were included in the baseline characteristics. Laboratory findings included hemoglobin, creatinine $(\mathrm{Cr})$, sodium, potassium, and total $\mathrm{CO}_{2}$. Intraoperative data included operation time, estimated blood loss (EBL) [16], and intraoperative hypotension. We used the code of the In-

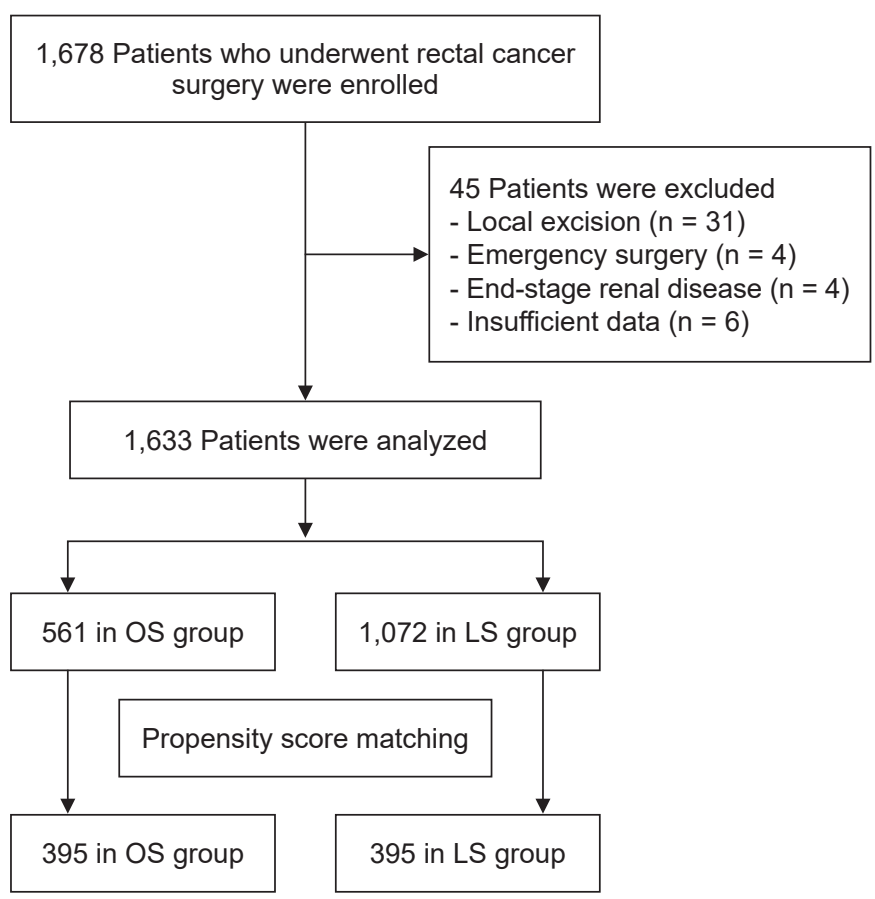

Figure 1. Flow chart of the study population.

LS, laparoscopic surgery; OS, open surgery. 
ternational Classification of Disease, 10th Revision to identify underlying comorbidities. Intraoperative hypotension was defined as systolic blood pressure of $\leq 90 \mathrm{mmHg}$ or use of inotropic agents such as dopamine, norepinephrine, or phenylephrine during the surgery. Operation time of $>240$ minutes was considered to be a long operation time [17]. Patients were considered to have low rectal cancer when the lower tumor margin was within $6 \mathrm{~cm}$ from the anal verge.

\section{Outcomes}

The primary objective of this study was to compare the incidence of postoperative AKI following LS and OS in patients with rectal cancer. AKI was defined as an absolute increase in serum $\mathrm{Cr}$ of $\geq 0.3 \mathrm{mg} / \mathrm{dL}$ over the baseline value or $\geq 1.5$ times higher than the baseline value, according to the serum $\mathrm{Cr}$ criteria in the Kidney Disease: Improving Global Outcome (KDIGO) guidelines [1]. We defined the baseline serum $\mathrm{Cr}$ value as the lowest serum $\mathrm{Cr}$ level measured $<90$ days before the surgery. We used the peak serum Cr level, which is the highest $\mathrm{Cr}$ value measured $<14$ days after surgery, to identify the stage of AKI according to the KDIGO criteria $[18,19]$. Severe AKI was defined as stage 2 or stage 3 AKI according to the KDIGO criteria. We also evaluated AKI recovery three months after postoperative AKI. We defined AKI recovery as a return of the serum $\mathrm{Cr}$ to a value less than 1.20 times the baseline serum Cr level [20]. The lowest serum $\mathrm{Cr}$ value measured $<90$ days after the AKI event was used. We also evaluated the 5 -year overall survival rate. As many mortality events occurred outside the study hospital, we reviewed the national death database of the Ministry of Interior and Security of Korea to identify the outcomes. Other outcomes included hospital stay, renal replacement therapy in-hospital days, and postoperative intensive care unit admission.

\section{Statistical analysis}

We applied propensity score matching analysis to minimize the influence of potential confounding biases and to increase comparability between the LS and OS groups. Among factors that can affect postoperative AKI, we included potentially explanatory variables that can be found through electronic medical records. The following variables were included to calculate the propensity scores using a multi- variate logistic regression model: age, sex, body mass index, ASA PS classification, neoadjuvant chemotherapy, previous operation history, tumor distance from anal verge, stage of cancer, hemoglobin, $\mathrm{Cr}$, sodium, potassium, total $\mathrm{CO}_{2}$, and operation year. A 1:1 propensity score matching method was applied based on the greedy 8-1-digit matching algorithm. Propensity score matching analysis was conducted using SAS version 9.1.3 (SAS Institute, Cary, NC, USA).

Mean \pm standard deviation was calculated for continuous variables. Categorical variables were reported as numbers and percentages of participants. The intergroup comparison of numerical data was performed using the Student $t$ test. The Pearson chi-square test was used to compare categorical data. The Cox proportional hazard regression analysis was performed to identify independent associations between the type of surgery and postoperative AKI in patients with rectal cancer. Survival curves were assessed using the Kaplan-Meier method and the statistical significance was estimated using the log-rank method. The additive interaction was analyzed using the relative excess risk due to interaction, attributable proportion due to interaction, and synergistic index [21] using R version 3.0.3 (R Foundation for Statistical Computing, Vienna, Austria). Statistical significance was set at a p-value of $<0.05$. With the exception of the propensity score matching analysis and additive interaction analysis, we performed all statistical analyses using IBM SPSS version 20 (IBM Corp., Armonk, NY, USA).

\section{Results}

A total of 1,633 patients were enrolled in the present study. The mean age of the entire cohort was $62.1 \pm 12.0$ years, and 1,039 patients (63.6\%) were males. Among these patients, $1,072(65.6 \%)$ and 561 (34.4\%) were included in the LS and OS groups, respectively. The baseline characteristics of the two groups are summarized in Table 1. In the entire cohort, the LS group had lower rates of hypertension, diabetes mellitus, previous operation history, and stage 3 or 4 cancer than the OS group. The distance of the tumor from the anal verge and levels of hemoglobin, sodium, potassium, and total $\mathrm{CO}_{2}$ were significantly higher in the LS group than in the OS group. The LS group had more recent cases than the OS group. After the propensity score matching, 395 patients remained in each group. The mean age of the matched patients was $61.9 \pm 12.2$, and 495 patients $(62.7 \%)$ were males. 
Table 1. Baseline characteristics of the patients

\begin{tabular}{|c|c|c|c|c|c|c|}
\hline \multirow[b]{2}{*}{ Parameter } & \multicolumn{3}{|c|}{ Entire cohort $(n=1,633)$} & \multicolumn{3}{|c|}{ Propensity-matched cohort $(n=790)$} \\
\hline & Open $(n=561)$ & $\begin{array}{c}\text { Laparoscopy } \\
(\mathrm{n}=1,072)\end{array}$ & p-value & Open $(n=395)$ & $\begin{array}{c}\text { Laparoscopy } \\
(\mathrm{n}=395)\end{array}$ & p-value \\
\hline Age (yr) & $62.2 \pm 12.2$ & $62.0 \pm 11.9$ & 0.69 & $62.4 \pm 12.2$ & $61.4 \pm 12.2$ & 0.25 \\
\hline Male sex & $349(62.2)$ & $690(64.4)$ & 0.39 & $257(65.1)$ & $238(60.3)$ & 0.16 \\
\hline Body mass index $\left(\mathrm{kg} / \mathrm{m}^{2}\right)$ & $23.1 \pm 3.4$ & $23.4 \pm 3.1$ & 0.06 & $23.2 \pm 3.4$ & $23.1 \pm 3.2$ & 0.77 \\
\hline Hypertension & $83(14.8)$ & $278(25.9)$ & $<0.001$ & $71(18.0)$ & $78(19.7)$ & 0.52 \\
\hline Diabetes mellitus & $47(8.4)$ & $141(13.2)$ & 0.004 & $44(11.1)$ & $40(10.1)$ & 0.64 \\
\hline Preoperative chemotherapy & $191(34.0)$ & $324(30.2)$ & 0.11 & $128(32.4)$ & $144(36.5)$ & 0.23 \\
\hline Previous operation history & $203(36.2)$ & $462(43.1)$ & 0.007 & $157(39.7)$ & $161(40.8)$ & 0.77 \\
\hline \multicolumn{7}{|l|}{ Cancer staging } \\
\hline 1,2 & $245(43.7)$ & $657(61.3)$ & $<0.001$ & $194(49.1)$ & $183(46.3)$ & 0.43 \\
\hline 3,4 & $316(56.3)$ & $415(38.7)$ & $<0.001$ & $201(50.9)$ & $212(53.7)$ & 0.43 \\
\hline Distance of tumor from AV (cm) & $6.0 \pm 3.2$ & $7.0 \pm 3.1$ & $<0.001$ & $6.4 \pm 3.2$ & $6.2 \pm 3.1$ & 0.19 \\
\hline ASA PS classification & $1.7 \pm 0.6$ & $1.7 \pm 0.6$ & 0.18 & $1.7 \pm 0.6$ & $1.7 \pm 0.6$ & 0.43 \\
\hline Hemoglobin (g/dL) & $12.4 \pm 2.0$ & $13.1 \pm 1.8$ & $<0.001$ & $12.7 \pm 2.0$ & $12.7 \pm 1.8$ & 0.73 \\
\hline Creatinine $(\mathrm{mg} / \mathrm{dL})$ & $1.1 \pm 5.5$ & $0.9 \pm 0.2$ & 0.21 & $0.9 \pm 0.2$ & $0.9 \pm 0.2$ & 0.15 \\
\hline Sodium (mmol/L) & $139.8 \pm 3.0$ & $140.5 \pm 2.7$ & $<0.001$ & $140.0 \pm 2.9$ & $140.0 \pm 2.8$ & 0.96 \\
\hline Potassium (mmol/L) & $4.1 \pm 0.4$ & $4.2 \pm 0.4$ & 0.003 & $4.2 \pm 0.4$ & $4.2 \pm 0.4$ & 0.88 \\
\hline Total $\mathrm{CO}_{2}(\mathrm{mmol} / \mathrm{L})$ & $24.7 \pm 2.8$ & $25.1 \pm 2.8$ & 0.007 & $24.7 \pm 2.7$ & $24.9 \pm 2.5$ & 0.31 \\
\hline Years of surgery (years from 2003) & $6.5 \pm 4.0$ & $9.1 \pm 3.4$ & $<0.001$ & $7.4 \pm 3.8$ & $7.7 \pm 3.6$ & 0.19 \\
\hline
\end{tabular}

Values are presented as mean \pm standard deviation or number (\%).

$\mathrm{AV}$, anal verge; ASA, American society of anesthesiologists; PS, physical status.

In the propensity-matched cohort, all the patient characteristics, including hypertension, diabetes mellitus, previous operation history, cancer staging, distance of tumor from anal verge, levels of hemoglobin, sodium, potassium, and total $\mathrm{CO}_{2}$, and year of surgery, were similar in the LS and OS groups.

Table 2 shows the intraoperative and postoperative variables of the two groups. The overall incidence of AKI after rectal cancer surgery was $12.1 \%$. Before propensity score matching, the LS group had lower rates of AKI, intensive care unit admission, postoperative transfusion, and overall mortality at 5 years than the OS group. Hospital stay, operation time, and EBL were lower in the LS group than in the OS group. Intraoperative hypotension was lower in the OS group than in the LS group. After matching, lower rates of AKI, intensive care unit admission, postoperative transfusion, and overall mortality at 5 years were observed in the LS group compared to the OS group. The LS group had shorter hospital stay and operation time and less EBL than the OS group. However, there were no significant differences in intraoperative hypotension between the two groups.

Cox proportional hazards analyses were performed to identify risk factors of postoperative AKI in patients with rectal cancer (Table 3). In crude analysis, operation time of more than 240 minutes, large volume of EBL, and postoperative transfusion were associated with an increased risk of AKI after rectal cancer, and LS was associated with a decreased risk of postoperative AKI. In the multivariable analysis, postoperative transfusion (hazard ratio [HR], 2.495; 95\% confidence interval [CI], 1.529-4.072; $\mathrm{p}<0.001$ ) was associated with an increased risk of AKI, and LS (HR, 0.615; 95\% CI, 0.403-0.936; $p=0.02$ ) was associated with reduced risk of postoperative $\mathrm{AKI}$ in patients with rectal cancer.

Since postoperative transfusion was lower in the LS group, we assessed the effects of the interaction between LS and postoperative transfusion on postoperative AKI (Table 4). Patients who underwent OS and received postoperative transfusion were at 4.548 -fold increased risk for postoperative AKI compared to patients who underwent LS and did not receive postoperative transfusion $(\mathrm{p}<0.001)$. However, there was no statistical significance in additive scale and multiplicative scale. We also performed a two-way analysis of variance (ANOVA) to analyze the interaction between LS and postoperative transfusion (Supplementary Table 1, 
Table 2. Comparison of intraoperative and postoperative parameters

\begin{tabular}{|c|c|c|c|c|c|c|}
\hline \multirow[b]{2}{*}{ Parameter } & \multicolumn{3}{|c|}{ Entire cohort $(n=1,633)$} & \multicolumn{3}{|c|}{ Propensity-matched cohort $(n=790)$} \\
\hline & Open $(n=561)$ & $\begin{array}{l}\text { Laparoscopy } \\
(\mathrm{n}=1,072)\end{array}$ & p-value & Open $(n=395)$ & $\begin{array}{l}\text { Laparoscopy } \\
\quad(\mathrm{n}=395)\end{array}$ & p-value \\
\hline AKI stage & $97 / 561(17.3)$ & $100 / 1,072(9.3)$ & $<0.001$ & 63/395 (15.9) & 39/395 (9.9) & 0.01 \\
\hline 1 & $87 / 97(89.7)$ & $87 / 100(87.0)$ & 0.56 & $55 / 63(87.3)$ & $35 / 39(89.7)$ & $>0.99$ \\
\hline 2 & 10/97 (10.3) & $12 / 100(12.0)$ & 0.71 & $8 / 63(12.7)$ & 4/39 (10.3) & $>0.99$ \\
\hline 3 & $0 / 97(0)$ & $1 / 100(1.0)$ & $>0.99$ & $0 / 63(0)$ & $0 / 39(0)$ & $>0.99$ \\
\hline Severe AKI & 10/97 (10.3) & $13 / 100(13.0)$ & 0.56 & $8 / 63(12.7)$ & 4/39 (10.3) & $>0.99$ \\
\hline AKI recovery & $46 / 97(47.4)$ & $51 / 100(51.0)$ & 0.62 & $32 / 63(50.8)$ & $20 / 39(51.3)$ & 0.96 \\
\hline RRT in hospital days & 4/561 (0.7) & $0 / 1,072(0)$ & 0.01 & 2/395 (0.5) & 0/395 (0) & 0.499 \\
\hline Hospital stay (day) & $19.3 \pm 15.3$ & $13.4 \pm 7.0$ & $<0.001$ & $18.3 \pm 13.6$ & $14.1 \pm 7.2$ & $<0.001$ \\
\hline ICU admission & $99 / 561(17.6)$ & $79 / 1,072(7.4)$ & $<0.001$ & 69/395 (17.5) & $31 / 395(7.8)$ & $<0.001$ \\
\hline Operation time (min) & $263.0 \pm 129.4$ & $225.5 \pm 77.2$ & $<0.001$ & $265.1 \pm 132.9$ & $236.9 \pm 79.8$ & $<0.001$ \\
\hline Estimated blood loss (mL) & $392.8 \pm 532.2$ & $146.9 \pm 158.9$ & $<0.001$ & $408.1 \pm 604.4$ & $175.7 \pm 195.3$ & $<0.001$ \\
\hline Intraoperative hypotension & $215 / 561(38.3)$ & $519 / 1,072(48.4)$ & $<0.001$ & $168 / 395(42.5)$ & $148 / 395(37.5)$ & 0.15 \\
\hline Postoperative transfusion & $80 / 561(14.3)$ & $50 / 1,072(4.7)$ & $<0.001$ & $56 / 395(14.2)$ & 24/395 (6.1) & $<0.001$ \\
\hline Overall mortality at 5 year & $185 / 561(33.0)$ & $113 / 1,072(10.5)$ & $<0.001$ & 113/395 (28.6) & 65/395 (16.5) & $<0.001$ \\
\hline Sodium (mmol/L) & $139.8 \pm 3.0$ & $140.5 \pm 2.7$ & $<0.001$ & $140.0 \pm 2.9$ & $140.0 \pm 2.8$ & 0.96 \\
\hline
\end{tabular}

Values are presented as number (\%) or mean \pm standard deviation.

$\mathrm{AKI}$, acute kidney injury; ICU, intensive care unit; RRT, renal replacement therapy.

Table 3. Cox proportional hazard model to identify risk factors of acute kidney injury after rectal cancer surgery in propensity-matched cohort

\begin{tabular}{|c|c|c|c|c|}
\hline \multirow{2}{*}{ Variable } & \multicolumn{2}{|c|}{ Univariable } & \multicolumn{2}{|c|}{ Multivariable } \\
\hline & $\operatorname{HR}(95 \% \mathrm{Cl})$ & $\mathrm{p}$-value & $\mathrm{HR}(95 \% \mathrm{Cl})$ & p-value \\
\hline Operation time $>240 \mathrm{~min}$ & $1.49(1.01-2.20)$ & 0.045 & $1.37(0.90-2.10)$ & 0.14 \\
\hline 1-mL incremental blood loss & $1.00(1.00-1.00)$ & 0.01 & $1.00(1.00-1.00)$ & 0.67 \\
\hline Postoperative transfusion & $2.66(1.66-4.27)$ & $<0.001$ & $2.50(1.53-4.07)$ & $<0.001$ \\
\hline Intraoperative hypotension & $1.42(0.96-2.09)$ & 0.08 & & \\
\hline Laparoscopic surgery & $0.60(0.40-0.89)$ & 0.01 & $0.62(0.40-0.94)$ & 0.02 \\
\hline
\end{tabular}

$\mathrm{HR}$, hazard ratio; $\mathrm{Cl}$, confidence interval.

Table 4. Interaction analysis between laparoscopic surgery and postoperative transfusion for postoperative acute kidney injury

\begin{tabular}{|c|c|c|c|c|c|}
\hline \multirow{3}{*}{ Laparoscopic surgery } & \multicolumn{4}{|c|}{ Postoperative transfusion } & \multirow{3}{*}{$\begin{array}{l}\text { OR }(95 \% \mathrm{Cl}) \text { for postoperative } \\
\text { transfusion (yes vs. no) within } \\
\text { strata of laparoscopic surgery }\end{array}$} \\
\hline & \multicolumn{2}{|r|}{ No } & \multicolumn{2}{|r|}{ Yes } & \\
\hline & Number $^{\mathrm{a}}$ & OR $(95 \% \mathrm{Cl})$ & Number $^{a}$ & OR $(95 \% \mathrm{Cl})$ & \\
\hline Yes & $35 / 336$ & 1.00 (reference) & $4 / 20$ & $1.92(0.62-5.94)$ & $1.92(0.62-5.94)$ \\
\hline No & $45 / 294$ & $1.47(0.92-2.35)$ & $18 / 38$ & $4.55(2.35-8.80)$ & $3.10(1.63-5.89)$ \\
\hline $\begin{array}{l}\text { OR ( } 95 \% \mathrm{Cl}) \text { for laparoscopic } \\
\text { surgery (yes vs. no) within strata } \\
\text { of postoperative transfusion }\end{array}$ & & $1.47(0.92-2.35)$ & & $2.37(0.71-7.95)$ & \\
\hline
\end{tabular}


available online). Two-way ANOVA showed a significant effect of LS and postoperative transfusion on postoperative AKI. However, there was no significant interaction effect between LS and postoperative transfusion $(\mathrm{p}=0.13)$.

Fig. 2 shows Kaplan-Meier curves for postoperative AKI and overall survival according to the type of surgery. The cumulative incidence of postoperative AKI was lower in the LS group than in the OS group before $(9.3 \%$ vs. $17.3 \%$, $\mathrm{p}<$ $0.001)$ and after $(9.9 \%$ vs. $15.9 \%, \mathrm{p}=0.01)$ propensity score matching. As compared with the OS group, the LS group had a higher 5-year survival rate in the entire cohort $(89.5 \%$ vs. $67.0 \%, \mathrm{p}<0.001)$ and the propensity-matched cohort $(83.5 \%$ vs. $71.4 \%, \mathrm{p}<0.001)$.

Fig. 3 depicts subgroup analyses for postoperative AKI among the propensity-matched patients in the LS and OS groups. Patients with middle or high rectal cancer had lower postoperative AKI risk than patients with low rectal cancer ( $\mathrm{p}$ for interaction $=0.05)$. Patients with older age $(>65$ years),
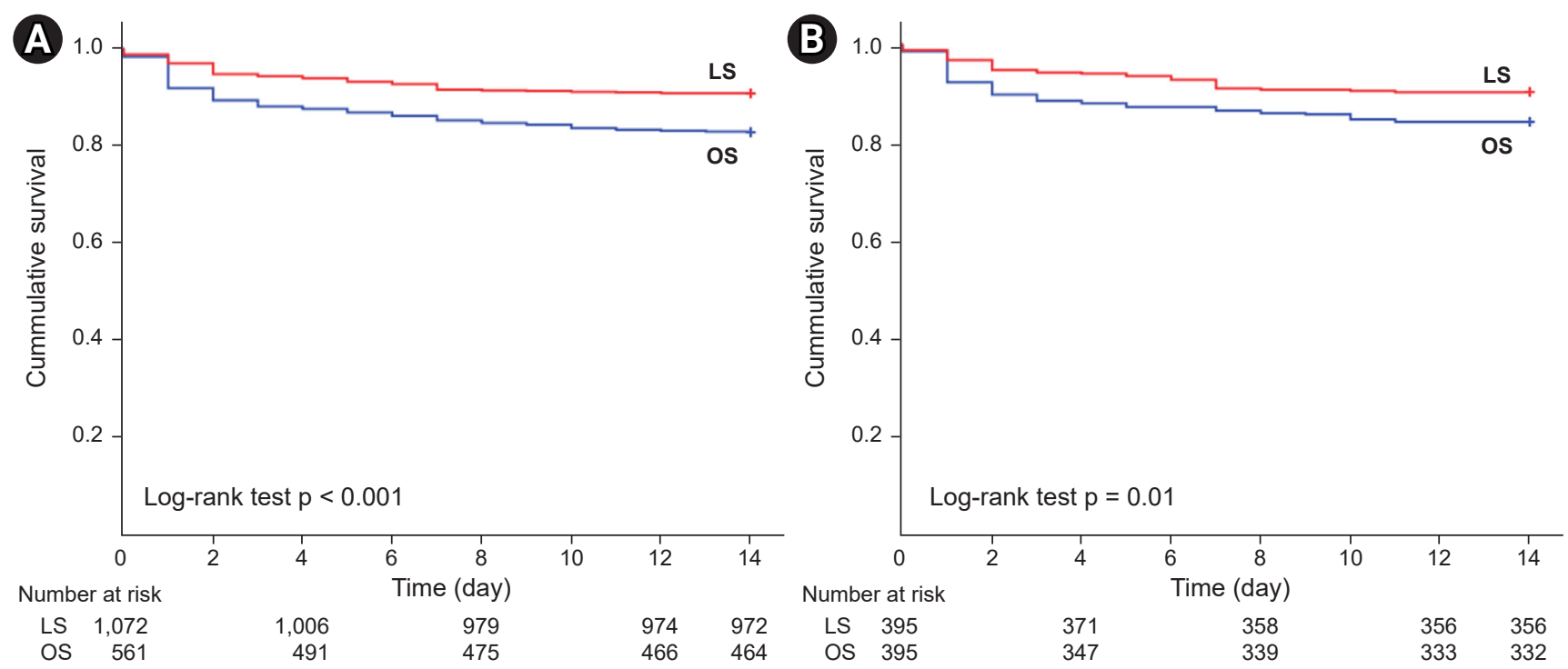
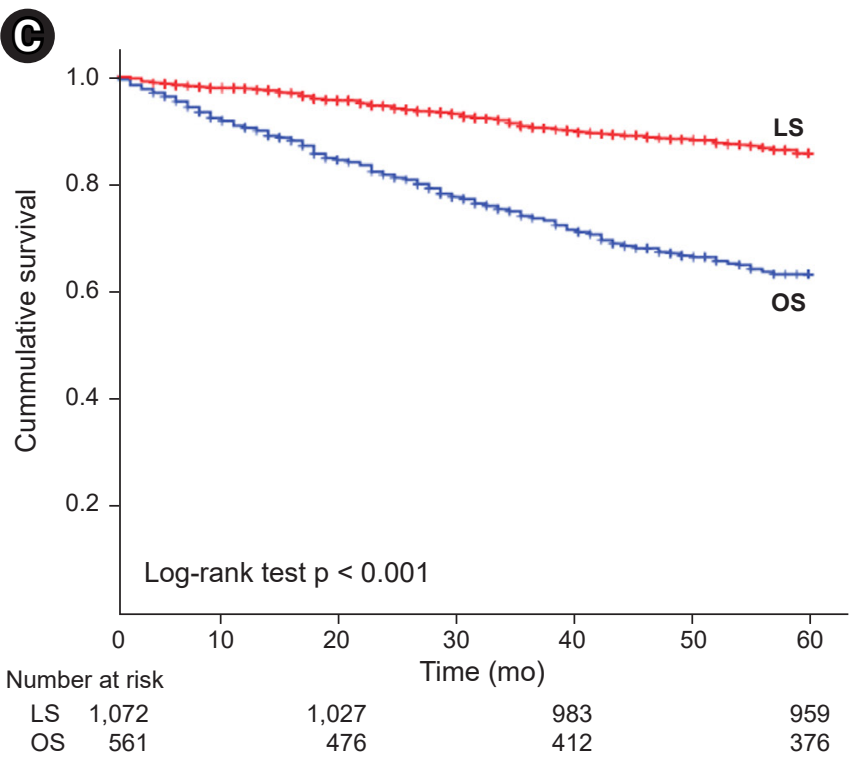

(D)

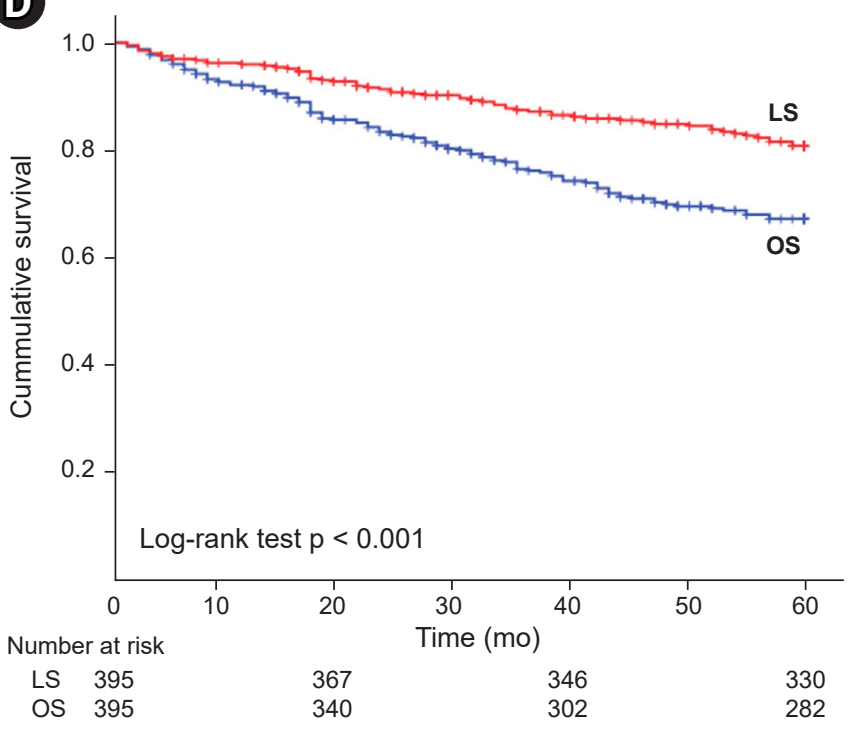

Figure 2. Kaplan-Meier curves for postoperative AKI and overall survival. (A) Postoperative AKI in the entire cohort. (B) Postoperative $\mathrm{AKI}$ in the propensity-matched cohort. (C) Overall survival in the entire cohort. (D) Overall survival in the propensity-matched cohort. AKI, acute kidney injury; LS, laparoscopic surgery; OS, open surgery. 


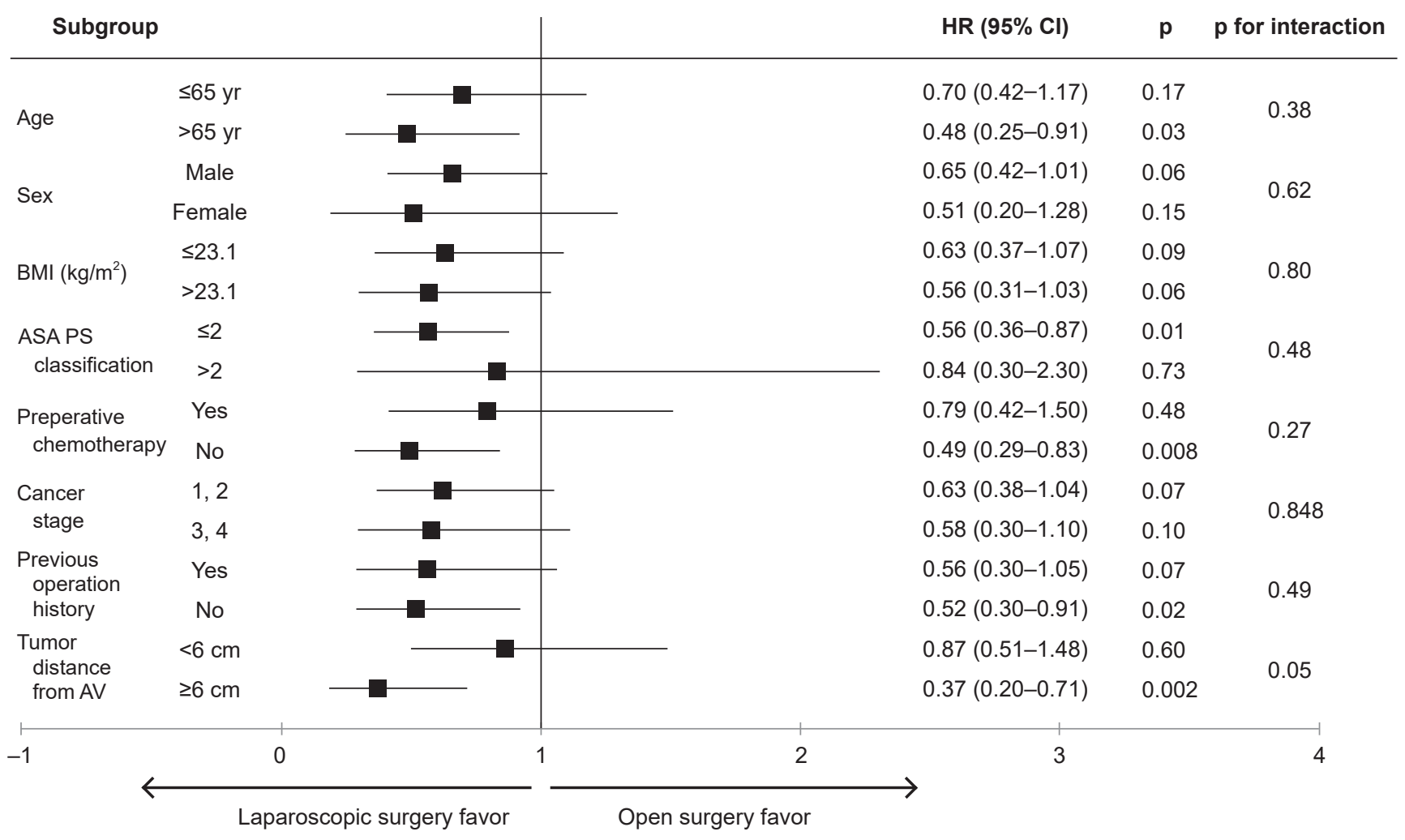

Figure 3. Cox proportional hazards analyses for postoperative acute kidney injury between laparoscopic surgery and open surgery in the propensity-matched cohort.

ASA, American Society of Anesthesiologists; AV, anal verge; BMI, body mass index; $\mathrm{Cl}$, confidence interval; HR, hazard ratio; PS, physical status.

lower ASA PS classification ( $\leq \mathrm{II})$, no preoperative chemotherapy, and no previous operation history demonstrated a lower postoperative AKI risk compared to their counterparts. However, there were no statistical significances on interactions.

\section{Discussion}

In the present study, we compared the incidence of postoperative AKI in rectal cancer patients after either LS or OS. The incidence of AKI after rectal cancer surgery was $12.1 \%$. We found that the rate of AKI after LS was significantly lower than after OS. This finding was consistent even after propensity score matching analysis. Operation time, EBL, and incidence of postoperative blood transfusion in the LS group were significantly lower than in the OS group. LS decreased the risk of postoperative AKI in the absence of blood transfusion. Among patients with middle or high rectal cancer, the LS group had a significantly lower incidence of postopera- tive AKI than the OS group.

In this study, the incidence of postoperative AKI in patients with rectal cancer was $12.1 \%$ in the entire cohort and $12.9 \%$ in the propensity-matched cohort. The incidence of AKI after rectal cancer surgery varies from $3.8 \%$ to $20.3 \%$ [22,23]. A cohort study including 288 rectal cancer patients revealed that the rate of postoperative AKI is $3.8 \%$ [22], and a population-based cohort study involving 1,337 patients reported an AKI incidence of $20.3 \%$ in patients with colorectal cancer [23]. These variations may be due to differences in type of cancer, definition of AKI, inclusion criteria, and exclusion criteria.

There are concerns about renal dysfunction resulting from increased intraabdominal pressure during LS. Hypercarbia due to $\mathrm{CO}_{2}$ insufflation has a chemical effect on cardiovascular changes. However, the $\mathrm{PaCO}_{2}$ levels usually observed during laparoscopy do not cause these complications [24]. Thus, increased intraabdominal pressure and related hormonal modifications contribute to most of the hemody- 
namic effects during laparoscopic procedures. Abdominal gas insufflation, which increases pressure on the kidney parenchyma and renal vessels, can lead to reduced cardiac output, blood flow, and urine output [25-27]. A previous animal study showed that when intraabdominal pressure was higher than $20 \mathrm{mmHg}$, venous pressure was increased and cardiac output was decreased. These changes led to a reduction in renal blood flow, and diminished urine output is observed [28]. A previous study involving 104 patients who underwent laparoscopic or open gastric bypass surgery found that urinary output during LS was significantly lower than during OS. However, postoperative blood urea nitrogen and Cr levels were not significantly different between the LS and OS groups. Increased renin-angiotensin-aldosterone system activity also contributes to renal dysfunction. In patients who underwent laparoscopic gastric bypass surgery, renal vasoconstriction and increased renin, aldosterone, and vasopressin levels were reported [29]. However, recent studies revealed that these changes do not increase the risk of AKI and that the incidence of postoperative AKI was even lower in LS patients compared to OS patients $[10,30]$. Our findings were consistent with those of recent trials.

Prolonged operation time may indicate complex surgical procedures that may directly or indirectly impair the kidney. Anemia and transfusion are established risk factors for AKI after cardiac surgery [31]. Anemia may aggravate kidney dysfunction by reducing kidney oxygen delivery, enhancing oxidative stress, and damaging hemostasis. Transfusion may worsen tissue oxygen delivery, encourage proinflammation, and promote tissue oxidative stress [32]. A previous study involving 1,340 patients who underwent robot-assisted laparoscopic radical prostatectomy or retropubic radical prostatectomy revealed that the EBL and red blood cell transfusion rate were significantly lower in the LS group compared to the OS group [30]. Another study involving 1,173 patients who underwent either laparoscopic liver resection or open liver resection showed that transfusion was an independent risk factor for postoperative AKI [10]. Our results support the findings of the aforementioned studies.

In multivariable analysis, LS and the lack of the need for postoperative blood transfusion were significantly associated with a decreased risk of postoperative AKI. Consistent with the findings of previous studies [10,30], blood transfusion was lower in the LS group in our study. We assumed that there was an effect of the interaction between LS and blood transfusion on postoperative AKI. However, there was no statistically significant interaction. During subgroup analyses, we demonstrated that patients with middle or high rectal cancer were at a lower risk for postoperative AKI compared to patients with low rectal cancer ( $\mathrm{p}$ for interaction $=0.05)$. Compared to middle or high rectal cancer surgery, low rectal cancer surgery is performed within a more confined space and requires more complex surgical procedures, which could be a plausible explanation for our findings following subgroup analyses. We assessed renal recovery in patients who developed AKI after rectal cancer surgery. However, there was no significant difference in renal recovery between the LS and OS groups. This study was retrospectively designed and there were no protocolized therapeutic interventions for postoperative AKI.

Since the laparoscopic resection of colon cancer was introduced in 1991, LS has become widely used and has progressively replaced OS for the treatment of colon cancer [33]. Previous studies provided sufficient evidence for the favorable outcome of LS in patients with colon cancer [34,35]. However, there is a lack of evidence for the benefits of LS in patients with rectal cancer. Although several studies have compared surgical and oncological outcomes between LS and OS in rectal cancer patients, the efficacy of the laparoscopic procedure is still controversial [36-38]. The ACOSOG Z6051 randomized clinical trial (RCT), a multicenter noninferiority randomized trial involving 486 stage II or III rectal cancer patients, demonstrated that LS failed to prove the noninferiority of pathologic outcomes compared to OS [37]. On the other hand, an RCT with 1,044 patients from 30 hospitals reported contradictory results [36]. There were no significant differences in locoregional recurrence and disease-free and overall survival between the LS and OS groups in patients with rectal cancer. A recent meta-analysis including five RCTs and seven non-RCTs provided evidence for the noninferiority of surgical outcomes following LS, compared to OS, for the treatment of rectal cancer [38]. However, the beneficial effects of the laparoscopic approach in the treatment of rectal cancer remain controversial. Moreover, the National Comprehensive Cancer Network guidelines do not recommend LS as the treatment of choice for rectal cancer [39]. In our study, the overall 5-year survival rate was higher in the LS group than in the OS group. A recent report revealed that the LS group showed a better 5 -year survival rate than the OS group (82.6\% vs. $76.6 \%, p<0.001)$ [40]. In our 
study, the differences in the 5-year survival rate according to type of surgery were larger. The LS group had lower rates of stage 3 or 4 cancer than the OS group (38.7\% vs. $56.3 \%$, p < $0.001)$. These differences may affect the result.

There have been few studies investigating the postoperative renal outcomes after rectal cancer surgery. A previous study involving 725 patients demonstrated that AKI was more common in the OS group than in the LS group. Another study including 5,420 patients also showed that postoperative AKI was significantly lower in the LS group compared to the OS group. However, both studies did not mainly focus on renal outcome. The first study did not define AKI and the second study did not compare with baseline $\mathrm{Cr}$ value. They did not identify risk factors for AKI and investigate renal recovery in patients with postoperative AKI. Our study has strength in analyzing the incidence of postoperative AKI and relevant factors.

The present study has some limitations. First, this cohort study is a single-center, retrospective study, and LS was not randomly performed on patients. Although we performed a propensity score matching analysis to minimize confounding biases, we could not collect all confounding factors that influenced our results. Therefore, the outcomes may be subject to unmeasured confounders. Second, even though we defined AKI using the KDIGO criteria, we could not evaluate the urine output. This study is a retrospective study. Therefore, it was impossible to measure the urine output of all study patients in the general ward. Thus, the absence of data on urine output may lead to the misclassification of AKI. Third, increased intraabdominal pressure is one of the main mechanisms that explain postoperative AKI after LS. However, we did not measure the intraabdominal pressure during surgery.

In conclusion, this large retrospective cohort study showed that the incidence of postoperative AKI was significantly lower in LS than in OS for rectal cancer surgery. During subgroup analyses, the LS group had a lower incidence of postoperative AKI than the OS group, especially among patients with middle or high rectal cancer. LS may have a positive effect on postoperative AKI in rectal cancer patients.

\section{Conflicts of interest}

All authors have no conflicts of interest to declare.

\section{Funding}

This research was supported by a grant No. 2019R1A2 C1085411 from the National Research Foundation, and by a grant of the Korea Health Technology R\&D Project through the Korea Health Industry Development Institute (KHIDI), funded by the Ministry of Health \& Welfare, Republic of Korea (grant number: HI17C1827).

\section{Authors' contributions}

Conceptualization: SIK, JR, SK, JCJ, HJC, KYN, DWC, SBK Investigation, Data curation: SIK, SYL, JYR, HES

Formal analysis: JHP

Funding acquisition: SK

Writing-original draft: JHP

Writing-review \& editing: SIK, JR, SK, JCJ, HJC, KYN, DWC, SBK

All authors read and approved the final manuscript.

\section{ORCID}

Jin Hyuk Paek, https://orcid.org/0000-0001-8875-1260 Sung Il Kang, https://orcid.org/0000-0002-5023-0168 Jiwon Ryu, https://orcid.org/0000-0001-7927-9654 Sung Yoon Lim, https://orcid.org/0000-0001-8875-1260 Ji Young Ryu, https://orcid.org/0000-0001-7686-3783 Hyung Eun Son, https://orcid.org/0000-0002-8719-3823 Jong Cheol Jeong, https://orcid.org/0000-0002-5024-2927 Ho Jun Chin, https://orcid.org/0000-0002-3710-0190 Ki Young Na, https://orcid.org/0000-0002-8872-8236 Dong-Wan Chae, https://orcid.org/0000-0001-9401-892X Sung-Bum Kang, https://orcid.org/0000-0002-9574-5069 Sejoong Kim, https://orcid.org/0000-0002-7238-9962

\section{References}

1. Kidney Disease: Improving Global Outcomes (KDIGO) Acute Kidney Injury Work Group. KDIGO Clinical Practice Guideline for Acute Kidney Injury. Kidney Int Suppl 2012;2:1-138.

2. Chertow GM, Burdick E, Honour M, Bonventre JV, Bates DW. Acute kidney injury, mortality, length of stay, and costs in hospitalized patients. J Am Soc Nephrol 2005;16:3365-3370.

3. Lameire NH, Bagga A, Cruz D, et al. Acute kidney injury: an increasing global concern. Lancet 2013;382:170-179. 
4. Thakar CV, Christianson A, Freyberg R, Almenoff P, Render ML. Incidence and outcomes of acute kidney injury in intensive care units: a Veterans Administration study. Crit Care Med 2009;37:2552-2558.

5. Uchino S, Kellum JA, Bellomo R, et al. Acute renal failure in critically ill patients: a multinational, multicenter study. JAMA 2005;294:813-818.

6. Thakar CV. Perioperative acute kidney injury. Adv Chronic Kidney Dis 2013;20:67-75.

7. Bihorac A, Yavas S, Subbiah S, et al. Long-term risk of mortality and acute kidney injury during hospitalization after major surgery. Ann Surg 2009;249:851-858.

8. Kim CS, Bae EH, Ma SK, Kweon SS, Kim SW. Impact of transient and persistent acute kidney injury on chronic kidney disease progression and mortality after gastric surgery for gastric cancer. PLoS One 2016;11:e0168119.

9. Veldkamp R, Kuhry E, Hop WC, et al. Laparoscopic surgery versus open surgery for colon cancer: short-term outcomes of a randomised trial. Lancet Oncol 2005;6:477-484.

10. Moon YJ, Jun IG, Kim KH, Kim SO, Song JG, Hwang GS. Comparison of acute kidney injury between open and laparoscopic liver resection: propensity score analysis. PLoS One 2017;12:e0186336.

11. Park YS, Jun IG, Go Y, Song JG, Hwang GS. Comparison of acute kidney injury between open and laparoscopic pylorus-preserving pancreaticoduodenectomy: propensity score analysis. PLoS One 2018;13:e0202980.

12. Quintana JM, Anton-Ladislao A, Lázaro S, et al. Outcomes of open versus laparoscopic surgery in patients with rectal cancer. Int J Colorectal Dis 2018;33:99-103.

13. Greenblatt DY, Rajamanickam V, Pugely AJ, Heise CP, Foley EF, Kennedy GD. Short-term outcomes after laparoscopic-assisted proctectomy for rectal cancer: results from the ACS NSQIP. J Am Coll Surg 2011;212:844-854.

14. Keys A, Fidanza F, Karvonen MJ, Kimura N, Taylor HL. Indices of relative weight and obesity. J Chronic Dis 1972;25:329-343.

15. Saklad M. Grading of patients for surgical procedures. Anesthesiology 1941;2:281-284.

16. Lenoir B, Merckx P, Paugam-Burtz C, et al. Individual probability of allogeneic erythrocyte transfusion in elective spine surgery: the predictive model of transfusion in spine surgery. Anesthesiology 2009;110:1050-1060.

17. Hobson C, Singhania G, Bihorac A. Acute kidney injury in the surgical patient. Crit Care Clin 2015;31:705-723.

18. Wehbe E, Brock R, Budev M, et al. Short-term and long-term out- comes of acute kidney injury after lung transplantation. J Heart Lung Transplant 2012;31:244-251.

19. Park S, Cho H, Park S, et al. Simple Postoperative AKI Risk (SPARK) Classification before noncardiac surgery: a prediction index development study with external validation. J Am Soc Nephrol 2019;30:170-181.

20. Park S, Baek SH, Ahn S, et al. Impact of electronic Acute Kidney Injury (AKI) alerts with automated nephrologist consultation on detection and severity of AKI: a quality improvement study. Am J Kidney Dis 2018;71:9-19.

21. Rothman KJ. Synergy and antagonism in cause-effect relationships. Am J Epidemiol 1974;99:385-388.

22. Lim SY, Lee JY, Yang JH, et al. Predictive factors of acute kidney injury in patients undergoing rectal surgery. Kidney Res Clin Pract 2016;35:160-164.

23. Slagelse C, Gammelager H, Iversen LH, Sørensen HT, Christiansen CF. Acute kidney injury and 1-year mortality after colorectal cancer surgery: a population-based cohort study. BMJ Open 2019;9:e024817.

24. Rasmussen JP, Dauchot PJ, DePalma RG, et al. Cardiac function and hypercarbia. Arch Surg 1978;113:1196-1200.

25. Dunn MD, McDougall EM. Renal physiology. Laparoscopic considerations. Urol Clin North Am 2000;27:609-614.

26. McDougall EM, Monk TG, Wolf JS Jr, et al. The effect of prolonged pneumoperitoneum on renal function in an animal model. J Am Coll Surg 1996;182:317-328.

27. Chang DT, Kirsch AJ, Sawczuk IS. Oliguria during laparoscopic surgery. J Endourol 1994;8:349-352.

28. Shuto K, Kitano S, Yoshida T, Bandoh T, Mitarai Y, Kobayashi M. Hemodynamic and arterial blood gas changes during carbon dioxide and helium pneumoperitoneum in pigs. Surg Endosc 1995;9:1173-1178.

29. Nguyen NT, Cronan M, Braley S, Rivers R, Wolfe BM. Duplex ultrasound assessment of femoral venous flow during laparoscopic and open gastric bypass. Surg Endosc 2003;17:285-290.

30. Joo EY, Moon YJ, Yoon SH, Chin JH, Hwang JH, Kim YK. Comparison of acute kidney injury after robot-assisted laparoscopic radical prostatectomy versus retropubic radical prostatectomy: a propensity score matching analysis. Medicine (Baltimore) 2016;95:e2650.

31. Karkouti K, Wijeysundera DN, Yau TM, et al. Acute kidney injury after cardiac surgery: focus on modifiable risk factors. Circulation 2009;119:495-502.

32. Almac E, Ince C. The impact of storage on red cell function in blood transfusion. Best Pract Res Clin Anaesthesiol 2007;21:195- 
208.

33. Jacobs M, Verdeja JC, Goldstein HS. Minimally invasive colon resection (laparoscopic colectomy). Surg Laparosc Endosc 1991;1: 144-150.

34. Colon Cancer Laparoscopic or Open Resection Study Group; Buunen M, Veldkamp R, et al. Survival after laparoscopic surgery versus open surgery for colon cancer: long-term outcome of a randomised clinical trial. Lancet Oncol 2009;10:44-52.

35. Lacy AM, García-Valdecasas JC, Delgado S, et al. Laparoscopy-assisted colectomy versus open colectomy for treatment of non-metastatic colon cancer: a randomised trial. Lancet 2002;359:2224-2229.

36. Bonjer HJ, Deijen CL, Abis GA, et al. A randomized trial of laparoscopic versus open surgery for rectal cancer. $N$ Engl J Med 2015;372:1324-1332.
37. Fleshman J, Branda M, Sargent DJ, et al. Effect of laparoscopic-assisted resection vs open resection of stage II or III rectal cancer on pathologic outcomes: the ACOSOG Z6051 randomized clinical trial. JAMA 2015;314:1346-1355.

38. Milone M, Manigrasso M, Burati M, Velotti N, Milone F, De Palma GD. Surgical resection for rectal cancer. Is laparoscopic surgery as successful as open approach? A systematic review with meta-analysis. PLoS One 2018;13:e0204887.

39. Benson AB, Venook AP, Al-Hawary MM, et al. Rectal cancer, version 2.2018, NCCN Clinical Practice Guidelines in Oncology. $J$ Natl Compr Canc Netw 2018;16:874-901.

40. Schnitzbauer V, Gerken M, Benz S, et al. Laparoscopic and open surgery in rectal cancer patients in Germany: short and longterm results of a large 10-year population-based cohort. Surg Endosc 2020;34:1132-1141. 